\title{
AVALIAÇÃO DO TRATAMENTO DA DISCOPATIA DEGENERATIVA CERVICAL PELA ARTRODESE VIA ANTERIOR UTILIZANDO PLACAS ASSOCIADAS A CAGES OU CAGES EM PEEK ISOLADAMENTE
}

\author{
EVALUATION OF CERVICAL DEGENERATIVE DISC DISEASE TREATMENT BY ANTERIOR \\ ARTHRODESIS USING PLATES ASSOCIATED WITH CAGES OR CAGES IN PEEK ALONE \\ EVALUACIÓN DEL TRATAMIENTO DE LA ENFERMEDAD DEGENERATIVA DEL DISCO \\ CERVICAL POR LA ARTRODESIS ANTERIOR UTILIZANDO PLACAS ASOCIADAS \\ CON JAULAS O JAULAS EN PEEC, AISLADAMENTE
}

André Rafael Hübner ${ }^{1}$, Marcelo Ribeiro Mendes² ${ }^{2}$ Jean Carlo Frigotto Queruz ${ }^{3}$, Jean Marcel Dambrós ${ }^{4}$ Álvaro Diego Heredia Suárez ${ }^{4}$, LeAndRo de Freitas SpinelLi ${ }^{5}$

\begin{abstract}
RESUMO
Objetivos: Avaliar comparativamente o tratamento da discopatia degenerativa cervical por discectomia e artrodese cervical via anterior utilizando placas associadas a cages ou cages em PEEK isoladamente. Métodos: Foi realizado um estudo retrospectivo comparativo entre dois grupos de pacientes operados pela técnica de discectomia e artrodese cervical via anterior. Foram selecionados aleatoriamente 70 pacientes, 35 operados com o método de fixação com placas associadas a cages - denominado Grupo I - e 35 com o cage em PEEK isoladamente - Grupo II. Realizou-se anamnese, exame físico, escores de dor (escala visual e analógica da dor) e função (critérios de Odom's, SF-36, Indice de incapacidade do pescoço) o pré e pós-operatório e exames de imagem. Resultados: Houve predominância de pacientes do sexo feminino em ambos os grupos, com média de idade de 55 anos no Grupo I e 47 no Grupo II. Ambos os grupos apresentaram distribuição semelhante quanto ao número de níveis operados, assim como nas complicações encontradas e escores de dor, cervicalgia e SF36 no pré e pós-operatório. Houve 97.1\% de fusão com 94.3\% de bons resultados no Grupo I e 100\% de fusão, com 97 \% de bons resultados no Grupo Il. Conclusões: O estudo comparativo da utilização de placas com cages e cages em PEEK isoladamente apresentou resultados semelhantes e satisfatórios para os grupos estudados, não se constatando superioridade ou inferioridade de um método com relação ao outro.
\end{abstract}

Descritores: Artrodese; Vértebras cervicais/cirurgia; Coluna/cirurgia.

\begin{abstract}
Objectives: To comparatively evaluate the treatment of cervical degenerative disc disease by anterior approach using plates associated or cages and PEEK device alone. Methods: A retrospective study comparing two groups of patients treated by anterior arthrodesis was performed. Seventy patients were randomly selected, 35 operated using plates associated with cages - Group I - and 35 with cages in PEEK alone - Group II. Medical history was obtained as well as physical examination, pain scores (pain visual analogue score) and function scores (Odom's criteria, SF-36, Neck Disability Index) and the pre and postoperative imaging studies. Results: There was a predominance of female patients in both groups, with a mean age of 55 years in Group I and 47 in Group II. Both groups showed a similar distribution of the number of levels operated, as well as the complications and pain scores, SF36 and neck disability index pre- and postoperatively. There was a fusion rate of $97.1 \%$ with 94.3\% of good results in Group I and 100\% of fusion, with 97\% of good results in Group II. Conclusions: The comparative study of arthrodesis using anterior approach with use of plates and cages and cages in PEEK alone had similar and satisfactory results for both groups, showing no superiority or inferiority of one method over another.
\end{abstract}

Keywords: Arthrodesis; Cervical vertebrae/surgery; Spine/surgery.

\section{RESUMEN}

Objetivos: Evaluar comparativamente el tratamiento de la enfermedad degenerativa del disco cervical por discectomía y artrodesis cervical vía anterior, utilizando placas asociadas con el uso de jaulas o estas en PEEK [Poliéster-Éter-Éter-Cetona], aisladamente. Métodos: fue realizado un estudio retrospectivo comparativo de dos grupos de pacientes tratados con la técnica de discectomía y artrodesis cervical vía anterior. Se seleccionaron al azar 70 pacientes, 35 operados por el método de fijación con placas asociadas a jaulas - Grupo I - y 35 con las jaulas en PEEK, aisladamente - Grupo II. Se recolectarondatos de historial clínico, examen físico, puntuaciones de dolor (escala visual y analógica del dolor-EVA) y función (criterios de Odom, SF-36, Índice de Discapacidad Cenical) del pre y posoperatorio y exámenes de imagen. Resultados: Se encontró un predominio de pacientes de sexo femenino en ambos grupos, con una edad promediode 55 años en el Grupo I y de 47 en el Grupo II. Ambos grupos mostraron una distribución similar en el número de niveles operados, así como de las complicaciones encontradas y de las puntuaciones de dolor, índice de discapacidad cervical y SF36 para el pre y posoperatorio.

1- Preceptor do Serviço de Cirurgia da Coluna do Instituto de Ortopedia eTraumatologia de Passo Fundo - RS, Brasil.

2- Estagiário (R4) de Serviço de Cirurgia de Coluna do Instituto de Ortopedia eTraumatologia de Passo Fundo, RS, Brasil.

3- Médico Residente (R1) do Instituto de Ortopedia eTraumatologia de Passo Fundo, RS, Brasil.

4- Estagiário (R4) de Serviço de Cirurgia de Coluna do Instituto de Ortopedia eTraumatologia de Passo Fundo, RS, Brasil.

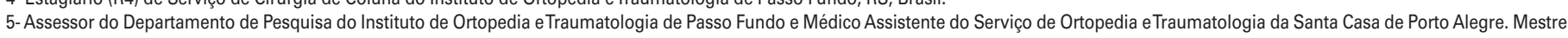
e Doutor pela Universidade Federal do Rio Grande do Sul. Brasil. 
Hubo 97,1\% para la fusión y 94,3\% de buenos resultados en el Grupo I y el 100\% de fusión, con 97\% de buenos resultados en el Grupo II. Conclusiones: El estudio comparativo de la utilización de placas con jaulas y estas en PEEK, aisladamente, presentó resultados similares y satisfactorios para los grupos estudiados, sin mostrar superioridad o inferioridad de un método sobre otro.

Descriptores: Artrodesis; Vértebras cervicales/cirugía; Columna/cirugía.

\section{INTRODUÇÃO}

A doença degenerativa do disco cervical tem etiologias variadas, podendo ter sua origem em condições diferentes como hérnia discal e espondilose. Os sintomas manifestam-se sob a forma de três síndromes álgicas: dor axial, dor radicular, mielopatia ou ainda com associação destas ${ }^{1-4}$. O envolvimento mais frequente ocorre na coluna cervical subaxial de C3 a C7 $7^{5-7}$ (Figura 1).

Os resultados do tratamento cirúrgico das doenças degenerativas da coluna cervical têm sido descrito por inúmeros autores. É consenso que a realização de discectomia e artrodese por via anterior para o tratamento da espondilose cervical demonstra, conforme descrito por Smith-Robinson, alta taxa de sucesso ao longo dos anos e, por isso, permanece como a principal opção cirúrgica. Os pacientes apresentam melhora clínica em até 90\% dos casos com o uso da técnica ${ }^{8,9}$

A utilização de placa cervical ainda está associada à ocorrência de outras complicações, como quebra ou soltura dos parafusos,

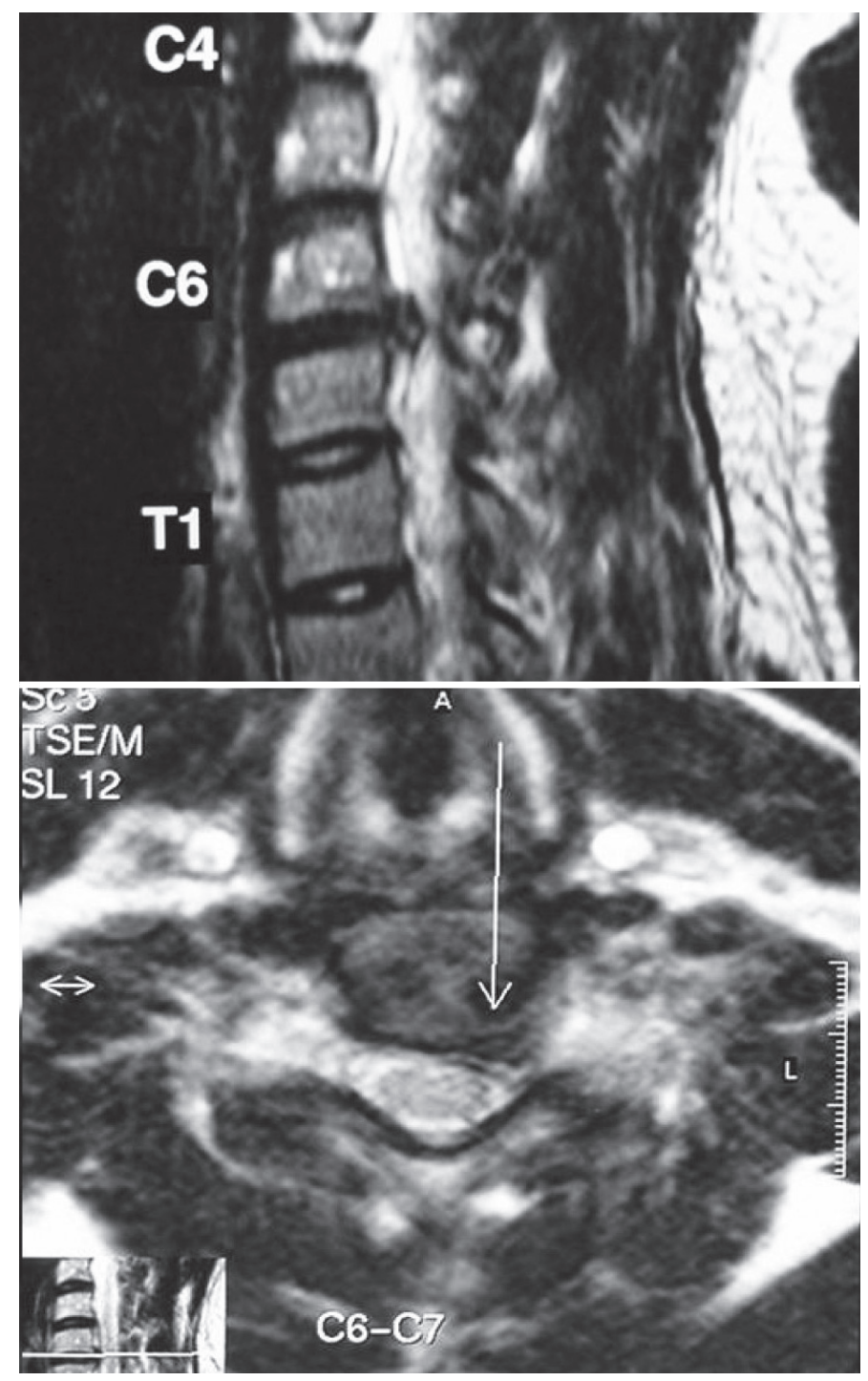

Figura 1. AMFS, 43 anos, sexo feminino, com cervicobraquialgia devido à hérnia discal cervical, nível C6-C7. À esquerda visualiza-se a hérnia em um corte sagital e à direita em um corte transversal em ressonância magnética. lesão esofágica ou lesão medular ou radicular por mau posicionamento dos implantes, além de tempo cirúrgico prolongado e aumento dos custos diretos da cirurgia ${ }^{10}$.

O dispositivo intersomático denominado cage foi desenvolvido para minimizar a morbidade da área doadora do enxerto. Através deste dispositivo é possível restaurar o suporte biomecânico imediato promovendo a osteointegração do enxerto que, utilizado em menor quantidade e na forma de osso esponjoso, tem a função de preencher o dispositivo. Desde 2000, a utilização do cage cervical tem se popularizado e, atualmente, ele é muito utilizado para a artrodese cervical. Existem cages de diversos materiais, porém os mais utilizados são os de titânio e os de PEEK (poli-éter-éter-cetona). Os resultados do procedimento são bons, com melhora significativa dos sintomas de dor e taxas de artrodese cervical superiores a 95\% na maioria das pesquisas científicas ${ }^{9,11}$. Existem poucos estudos que mostram os resultados da utilização de cage cervical isolado, seja em PEEK ou titânio, nas discectomias e artrodeses de três ou mais níveis. A maioria deles relata bons resultados, tanto clínicos quanto radiológicos, com taxas de consolidação acima de 90\% para três ou mais níveis ${ }^{12}$. O sucesso na utilização do cage seja isolado ou associado à placa em artrodeses cervicais se traduz pelo aumento de sua utilização atualmente ${ }^{13-19}$

A presente pesquisa avalia comparativamente o tratamento da discopatia degenerativa cervical por discectomia e artrodese cervical via anterior utilizando placa associada a cage ou cage em PEEK isoladamente.

\section{MATERIAIS E MÉTODOS}

A pesquisa envolve um estudo retrospectivo comparativo entre dois grupos de pacientes operados pela técnica de discectomia e artrodese cervical via anterior no Serviço de Cirurgia da Coluna do Instituto de Ortopedia e Traumatologia de Passo Fundo/RS. O estudo envolve 70 pacientes operados pela técnica e acometidos por discopatia degenerativa cervical. Foram selecionados aleatoriamente 35 pacientes em que foi utilizado como método de fixação o uso de placa associada a cage - denominado de Grupo I - e 35 pacientes em que se utilizou cage em PEEK isoladamente

- Grupo II (Figuras 2 e 3). Considerou-se um seguimento mínimo de seis meses, tempo este necessário para que houvesse fusão. Entretanto, a imensa maioria dos pacientes selecionados, mesmo de forma aleatória, apresenta um seguimento superior a um ano.

Todos os pacientes foram operados com a mesma técnica cirúrgica: incisão de Smith-Robinson; discectomia e descompressão até visualização do ligamento longitudinal posterior com a abertura do mesmo; remoção de osteófitos posteriores quando presentes e da hérnia discal; e preservação da placa vertebral óssea dos platôs superiores e inferiores abordados. Os cages utilizados nos dois grupos foram de PEEK, preenchidos com enxerto ósseo autólogo esponjoso de ilíaco.

Os dados dos pacientes foram coletados via prontuário médico no que se refere à anamnese, exame físico, escores de dor (escala visual e analógica da dor) e função (critérios de Odom's para a melhora dos sintomas (1: excelente; 2: bom; 3: regular; 4: ruim), SF-36 e o escore de Cervicalgia - Índice de incapacidade do pescoço) do pré e pós-operatórios. Da mesma maneira, foram avaliadas as radiografias (anteroposterior, perfil e dinâmicos) nos diversos momentos desde o pré-operatório até o seguimento mínimo considerado neste estudo. Foram avaliados listese estática e dinâmica, fusão, lise, alinhamento, quebra ou soltura do material. Quanto às complicações clínicas no pós-operatório, foram consideradas infecção superficial e profunda na ferida operatória cervical e no local de retirada de enxerto. 

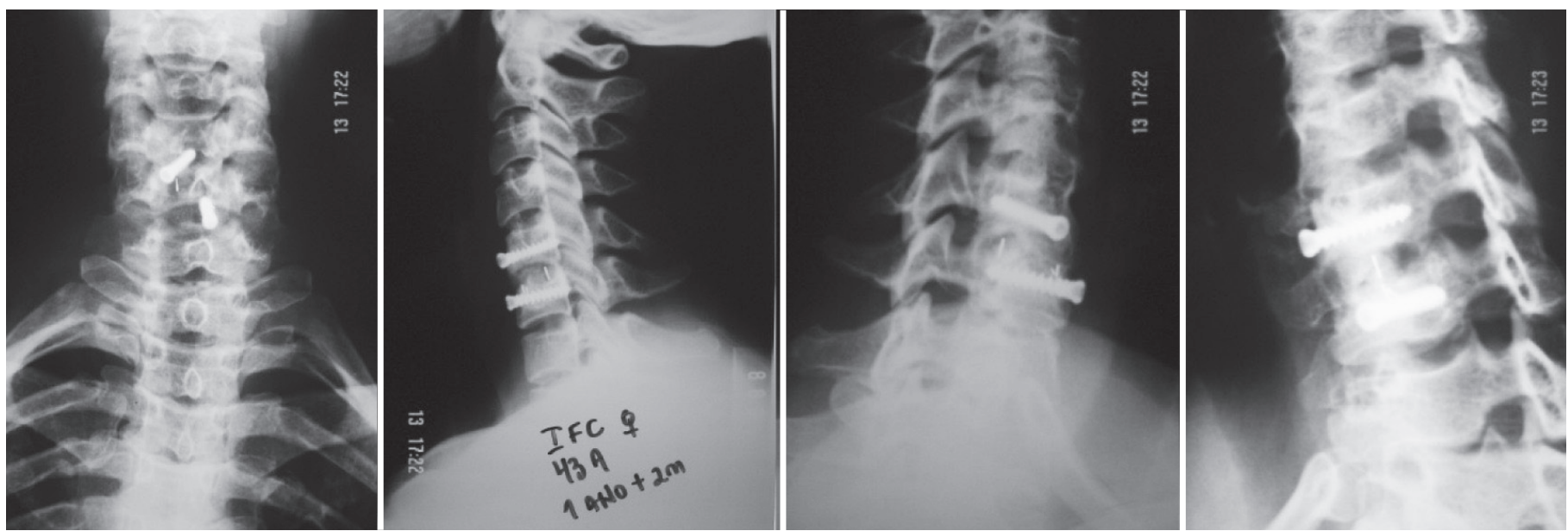

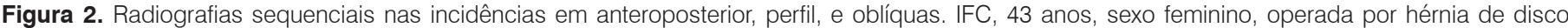
cervical de nível C5-C6 à direita com o uso de placa (radiotransparente) associada a cage.
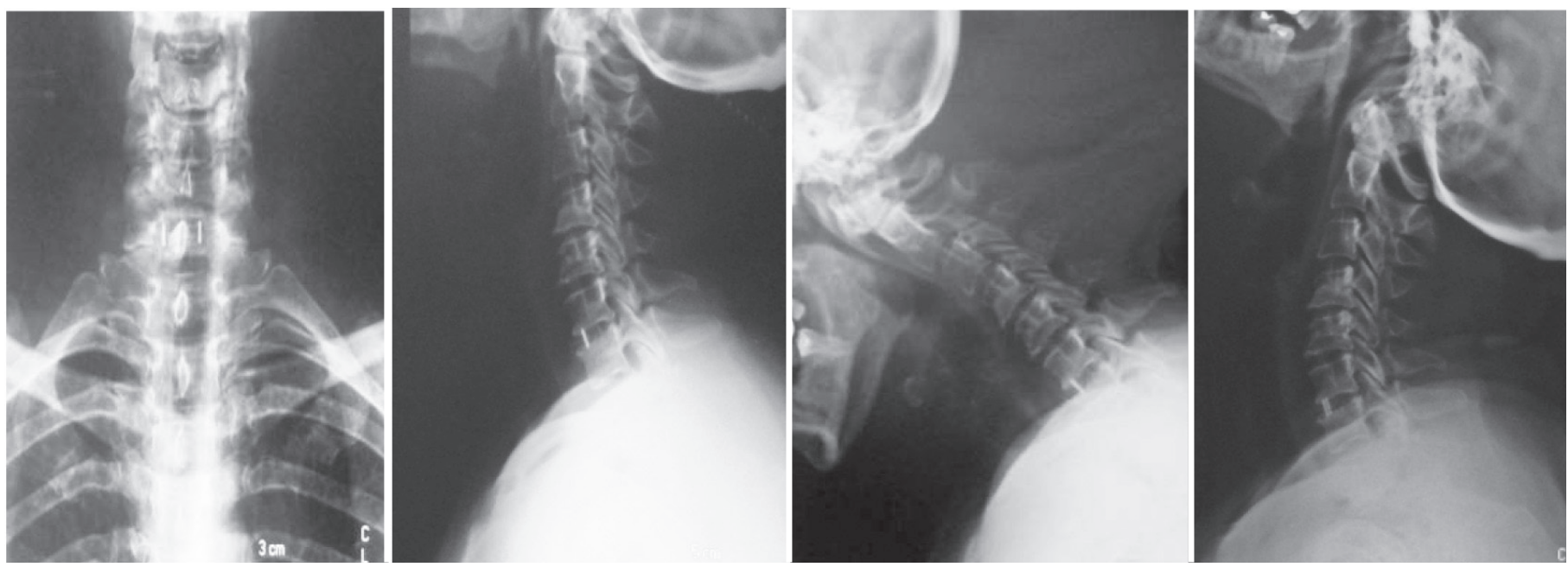

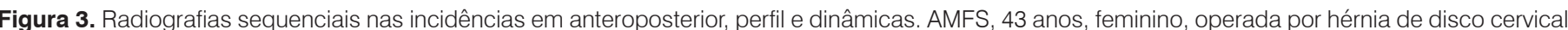
de nível C6-C7. No momento desta radiografia, apresentava seguimento de 3 meses pós-artrodese com cage em PEEK isolado (Solis).

Os critérios de inclusão foram os pacientes acometidos por doença cervical degenerativa e operados pela técnica de discectomia e artrodese cervical via anterior que utilizaram placa associado à cage ou cage em PEEK isoladamente, pacientes que não apresentavam cirurgias prévias cervicais e seguimento mínimo de seis meses. Os critérios de exclusão foram os pacientes com lesões associadas (fraturas, luxações e tumores) e pacientes com doenças sistêmicas que comprometessem a região cervical ou o seu resultado cirúrgico (artrite reumatóide, lúpus eritematoso sistêmico e diabetes).

A análise estatística dos dados for realizada com a utilização do programa SPSS para Windows v14 (SPSS Inc, Chicago, Illinois, USA). A significância estatística foi considerada quando $\mathrm{p}<0,05$. Foi considerada estatística descritiva, distribuição de frequências, teste de homogeneidade, teste $t$, Mann-Whitney ou qui-quadrado para a comparação entre os grupos, conforme o caso.

\section{RESULTADOS}

Entre os 35 pacientes selecionados do Grupo I (placa associada a cage), foram observados 20 pacientes $(57,1 \%)$ do sexo feminino e 15 (42,9\%) do sexo masculino, com um seguimento médio de 50 meses. A média de idade foi de 55 anos, variando de 37 a 71 anos. O tempo médio de internação hospitalar foi de dois dias. No Grupo II (Cage em PEEK isolado), foram avaliados 24 pacientes $(68,6 \%)$ do sexo feminino e $11(31,4 \%)$ do sexo masculino, com seguimento médio de 19 meses. A média de idade foi de 47 anos, variando de 37 a 67 anos. O tempo médio de internação hospitalar também foi de três dias.

Considerando-se os níveis operados, no Grupo I 22 pacientes (62.9\%) apresentavam acometimento de um nível, 13 (37.1\%) em dois níveis. Neste grupo, houve $97.1 \%$ de fusão com $94.3 \%$ de bons resultados. No Grupo II observou-se 24 pacientes (68.6\%) com acometimento de um nível, nove (25.7\%) com dois níveis e dois (5.7\%) com três níveis. Neste grupo observou-se 100\% de fusão, com $97 \%$ de bons resultados. A figura 4 apresenta exemplos de radiografias de um seguimento superior a um ano para ambos os grupos, mostrando a fusão.

Como complicações no Grupo I, observou-se a quebra da placa em um dos pacientes, ocorrendo posteriormente pseudoartrose. O paciente necessitou de um novo procedimento cirúrgico. Foram observados ainda dois casos de hematoma e dois casos de infecção superficial no local de retirada do enxerto. Todos os casos foram resolvidos com tratamento adequado (drenagem dos hematomas e antibioticoterapia com cefalexina no caso das infecções). Houve ainda dois pacientes que apresentaram dor persistente na área doadora do enxerto ósseo do ilíaco, que melhorou após seis meses da cirurgia. Não foi observado nenhum caso de disfagia ou de lesão do nervo laríngeo recorrente. No Grupo II houve dois casos de hematoma e um caso de infecção superficial do local de retirada do enxerto, também resolvidos após tratamento adequado (drenagem e antibioticoterapia, respectivamente). Não se observou 

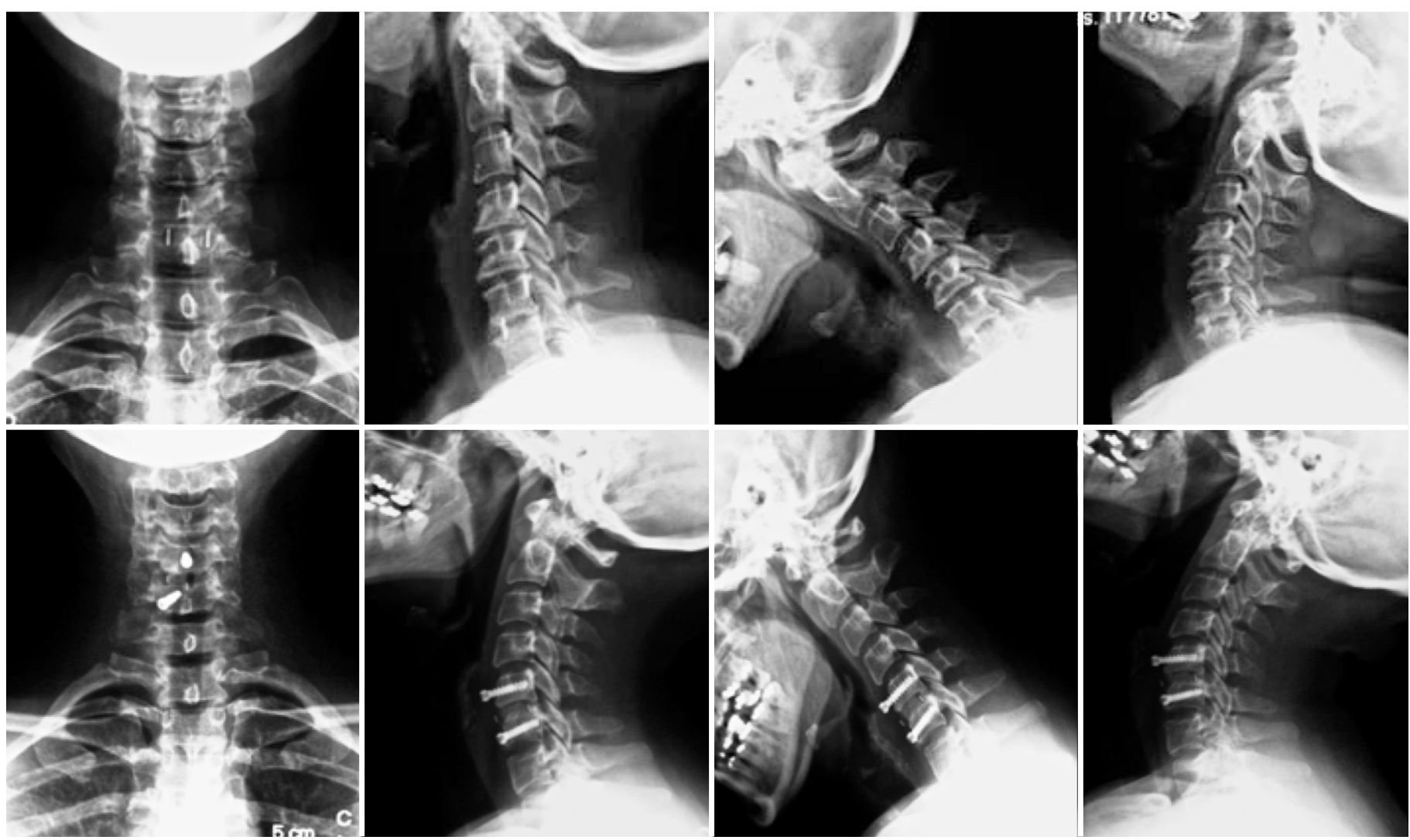

Figura 4. Resultados funcionais e visualização da fusão em pacientes dos Grupos I e II. Sequências nas incidências anteroposterior, perfil e dinâmicos Na linha superior, observa-se um paciente submetido à artrodese com cage isoladamente e,na linha inferior, um paciente com cage e placa.

casos de revisão cirúrgica por pseudoartrose, soltura ou migração de cage, listese ou lesão do laríngeo recorrente. Em nenhum dos grupos foram observados casos de infecção profunda.

O tempo de duração dos sintomas até o momento da cirurgia foi em média 11 meses para os Grupos I e II. Valores semelhantes foram observados quando se avaliou a escala visual e analógica de dor (EVA) no pré-operatório, com média de 78.3 no Grupo I e 77,7 no Grupo II. No pós-operatório, foi registrada uma EVA média de 10,9 no Grupo I e 10,3 no Grupo II. Não houve diferença estatisticamente significativa $(p>0,05)$ quanto à dor entre os grupos. Após quatro meses em média de pós-operatório, observou-se que 97,1\% dos cages no Grupo I e 100\% dos cages no Grupo II estavam integrados às vértebras, fato este comprovado pelo estudo radiográfico dinâmico em perfil da coluna cervical, bem como pela ausência de sinais de reabsorção óssea dos platôs adjacentes. Foi possível observar o adequado crescimento ósseo nos implantes em PEEK, devido a sua radiotransparência.

Todos os pacientes com algum grau de déficit neurológico inicial apresentaram melhora significativa dos sintomas, mesmo nos casos em que não houve a erradicação da dor. Um paciente do Grupo II persistiu com dor irradiada e dois outros com dor axial. No Grupo I, três pacientes permaneceram com dor axial, mas todos em menor intensidade do que no pré-operatório. Com relação aos protocolos SF36, no Grupo II houve um incremento de 96,8 para 121,8 e no questionário de cervicalgia houve melhora de 27 para 4,4, enquanto que no Grupo I o SF36 variou de 70,6 para 122,2 e no questionário de cervicalgia de 32,4 para 3,8. Não há diferença estatisticamente significativa $(p>0,05)$ entre os grupos.

\section{DISCUSSÃO}

Um disco degenerado pode gerar dor de varias maneiras, ocasionando estiramento ou compressão de nervo sinovertebral e terminações nervosas mais finas, alteração ou lesão de áreas inervadas, compressão de raiz, compressão central com disfunção neurológica ${ }^{20}$. A radiculopatia da hérnia discal na maioria das vezes melhora com tratamento conservador, enquanto a mielopatia espondilótica costuma ser progressiva. Por isso, quando o tratamento conservador da hérnia discal falha, pode-se indicar o tratamento cirúrgico. Estudos sobre a história natural da dor cervical e da radiculopatia espondilótica cervical com acompanhamento de 10 anos comprovam que ao longo dos anos, $79 \%$ dos pacientes apresentaram redução da dor, $43 \%$ obtiveram alívio quase completo dos sintomas, sendo que um terço do grupo estudado continuou com dor persistente; outras pesquisas mostram que $30 \%$ dos pacientes mantêm sintomas intermitentes e 25\% sintomas persistentes e em até $50 \%$ desses casos os sintomas podem persistir ao longo de 15 anos $^{21-24}$. Portanto, o consenso que existe é que o tratamento conservador melhora os sintomas radiculares em curto prazo, mas em longo prazo os sintomas costumam retornar.

Na história natural da mielopatia espondilótica cervical, 75\% dos pacientes tem agravamento dos sintomas, 20\% têm evolução lenta e progressiva e 5\% tem início e progressão rápida. O consenso é de que a maioria dos pacientes tenha progressão dos sintomas com períodos de estabilização ${ }^{25-28}$. A progressão não costuma acarretar incapacidade, mas existe deterioração neurológica com agravamento de funções de membros superiores e inferiores e quanto maior o agravamento e progressão, menor é a chance de cura e recuperação do paciente. Whitecloud e Seago $^{29}$ e Connor e Darden ${ }^{30}$ demonstraram bons e excelentes resultados em 70\% dos pacientes com artrodese intersomàtica anterior (para cervicalgia com discografia positiva). Palit et al. ${ }^{31}$ demonstraram que 79\% dos pacientes ficaram satisfeitos com o tratamento a longo prazo. Neste estudo observamos um tempo médio de 11 meses entre o aparecimento dos sintomas de mielopatia, cervicalgia ou radiculopatia e o procedimento cirúrgico. A partir dos critérios de Odom's, observamos que $94,3 \%$ dos pacientes apresentaram resultados excelentes ou bons no Grupo I e 97,1\% no Grupo II, sem diferença estatisticamente significativa $(p>0,05)$ entre os grupos. 
A indicação do procedimento cirúrgico ocorreu quando a dor cervical tinha origem discogênica e o paciente apresentava história de radiculopatia cervical com fracasso do tratamento conservador por três meses e nos pacientes com déficit neurológico progressivo $^{32}$. Na mielopatia cervical as indicações ainda não são bem definidas ${ }^{25}$. Neste trabalho, indicou-se o procedimento cirúrgico nos casos de mielopatia progressiva, grave ou moderada (com o paciente apresentando sintomas há menos de um ano) e branda que afetava suas atividades de rotina.

Encontramos apenas um caso de pseudoartrose, devido a quebra da placa, no Grupo I. O paciente foi submetido à reintervenção e apresentou fusão posteriormente. A maioria dos autores recomenda que na artrodese de múltiplos níveis seja adicionada estabilização com placa cervical anterior ou posterior, para minimizar a taxa de pseudoartrose ${ }^{10}$. Van Jonbergen et al. ${ }^{33}$ mostram que o seguimento de seis meses é suficiente para que haja a fusão óssea do seguimento operado. Considerando o total de 70 pacientes deste estudo, apenas 1,4\% apresentaram falha de fusão.

A discectomia cervical e artrodese via anterior com enxerto ósseo autólogo de ilíaco é o tratamento padrão-ouro para hérnia discal cervica ${ }^{34,35}$. Entretanto, os enxertos autólogos obtidos da crista ilíaca estão associados com morbidade do sítio doador e com- plicações como infecção superficial e profunda, fratura do ilíaco, formação de hematomas, deformidades palpáveis e lesão de ramos nervosos locais, que podem desencadear a meralgia parestésica - condição muito dolorosa, de difícil tratamento e que pode prejudicar o resultado clínico da cirurgia cervical ${ }^{11}$. Outra complicação da utilização de blocos de enxerto tricortical de ilíaco é a perda de altura do mesmo, com colapso do espaço discal e consolidação em cifose (subsidence) ${ }^{33}$. Por este motivo, não utilizamos em nossa prática blocos de enxerto diretamente. Entretanto, tivemos alguns casos de dor no local de retirada do enxerto, hematoma e infecção superficial. Não encontramos nesta série lesão nervosa decorrente do procedimento cirúrgico.

\section{CONCLUSÃO}

O estudo comparativo da utilização de placa associado a cage e cage em PEEK isoladamente apresentou resultados semelhantes e satisfatórios para os grupos estudados. Ambas as formas de artrodese se mostraram seguras, promovendo bons resultados clínicos e radiológicos. Sendo assim, representam uma boa alternativa de tratamento cirúrgico, não apresentando superioridade ou inferioridade de um método em relação ao outro.

\section{REFERÊNCIAS}

1. Clark CR. Cervical spondylotic myelopathy: history and physical findings. Spine (Phila Pa 1976). 1988;13(7):847-9.

2. Law MD Jr, Bernhardt M, White AA 3rd. Evaluation and management of cervical spondylotic myelopathy. Instr Course Lect. 1995;44:99-110.

3. Ono K, Ebara S, Fuji T, Yonenobu K, Fujiwara K, Yamashita K. Myelopathy hand. New clinical signs of cervical cord damage. J Bone Joint Surg Br. 1987;69(2):215-9.

4. Connell MD, Wiesel SW. Natural history and pathogenesis of cervical disk disease. Orthop Clin North Am. 1992;23(3):369-80.

5. LestiniWF, Wiesel SW. The pathogenesis of cervical spondylosis. Clin Orthop Relat Res. 1989;(239):69-93

6. Montgomery DM, Brower RS. Cervical spondylotic myelopathy. Clinical syndrome and natural history. Orthop Clin North Am. 1992;23(3):487-93.

7. Nurick S. The pathogenesis of the spinal cord disorder associated with cervical spondylosis. Brain. 1972:95(1):87-100

8. Hilibrand AS, Robbins M. Adjacent segment degeneration and adjacent segment disease: the consequences of spinal fusion? Spine J. 2004;4(6 Suppl):190S-194S.

9. Cho DY, Lee WY, Sheu PC. Treatment of multilevel cervical fusion with cages. Surg Neurol. 2004:62(5):378-85.

10. Hee HT, Majd ME, Holt RT, WhitecloudTS 3rd, Pienkowski D. Complications of multilevel cervical corpectomies and reconstruction with titanium cages and anterior plating. $J$ Spinal Disord Tech. 2003;16(1):1-8.

11. Rao RD, Currier BL, Albert TJ, Bono CM, Marawar SV, Poelstra KA, et al. Degenerative cervical spondylosis: clinical syndromes, pathogenesis, and management. J Bone Joint Surg Am. 2007:89(6):1360-78.

12. Moreland DB, Asch HL, Clabeaux DE, Castiglia GJ, Czajka GA, Lewis PJ, et al. Anterior cervical discectomy and fusion with implantable titanium cage: initial impressions, patient outcomes and comparison to fusion with allograft. Spine J. 2004;4(3):184-91.

13. Riley $L H J$ Jr, Robinson RA, Johnson KA, Walker AE. The results of anterior interbody fusion of the cervical spine. Review of ninety-three consecutive cases. J Neurosurg. 1969;30(2):127-33.

14. Robinson $R$, Walker $A R$, Ferlic $D C$, Wiecking $D K$. The results of anterior interbody fusion of the cervical spine. J Bone Joint Surg Am. 1962;44(8):1569-87.

15. Simpson J, An H. Degenerative disc disease of the cervical spine. In: An H, Simpson J. Surgery of the cervical spine. Baltimore: Williams and Wilkins. 1994. p.181-226.

16. White AA 3rd, Southwick WO, Deponte RJ, Gainor JW, Hardy R. Relief of pain by anterior cervical-spine fusion for spondylosis. A report of sixty-five patients. J Bone Joint Surg Am. 1973;55(3):525-34.

17. Yamamoto I, Ikeda A, Shibuya N, Tsugane R, Sato O. Clinical long-term results of anterior discectomy without interbody fusion for cervical disc disease. Spine (Phila Pa 1976). 1991;16(3):272-9.

18. ZdeblickTA, Bohlman HH. Cervical kyphosis and myelopathy. Treatment by anterior corpectomy and strut-grafting. J Bone Joint Surg Am. 1989;71(2):170-82.

19. Zhang ZH, Yin H, Yang K, Zhang T, Dong F, Dang G, Lou SQ, Cai Q. Anterior intervertebral disc excision and bone grafting in cervical spondylotic myelopathy. Spine (Phila Pa 1976). 1983:8(1):16-9.

20. Parke WW. Correlative anatomy of cervical spondylotic myelopathy. Spine (Phila Pa 1976). 1988;13(7):831-7.

21. Fishgrund J, Herkowitz $\mathrm{H}$. Anterior surgical procedures for cervical espondylotic radiculopathy and mielopathy. In: An H, Simpson J. Surgery of the cervical spine. Baltimore: Williams and Wilkins. 1994. p.195.

22. Gore DR, Sepic SB, Gardner GM, Murray MP. Neck pain: a long-term follow-up of 205 patients. Spine (Phila Pa 1976). 1987:12(1):1-5

23. Isomi T, Panjabi MM, Wang JL, Vaccaro AR, Garfin SR, PatelT. Stabilizing potential of anterior cervical plates in multilevel corpectomies. Spine (Phila Pa 1976). 1999;24(21):2219-23.

24. Less F, Turner JW. Natural history and prognosis of cervical spondylosis. Br Med J. 1963;2(5373):1607-10.

25. Bohlman HH. Cervical spondylosis and myelopathy. Instr Course Lect. 1995;44:81-97.

26. Bohlman HH, Emery SE. The pathophysiology of cervical spondylosis and myelopathy. Spine (Phila Pa 1976). 1988;13(7):843-6.

27. Crandall PH, Batzdorf U. Cervical spondylotic myelopathy. J Neurosurg. 1966;25(1):57-66.

28. LaRocca H. Cervical spondylotic myelopathy: natural history. Spine (Phila Pa 1976). 1988;13(7):854-5

29. Whitecloud TS 3rd, Seago RA. Cervical discogenic syndrome. Results of operative intervention in patients with positive discography. Spine (Phila Pa 1976). 1987:12(4):313-6.

30. Connor PM, Darden BV 2nd. Cervical discography complications and clinical efficacy. Spine (Phila Pa 1976). 1993;18(14):2035-8.

31. Palit M, Schofferman J, Goldthwaite N, Reynolds J, Kerner M, Keaney D, et al. Anterior discectomy and fusion for the management of neck pain. Spine (Phila Pa 1976). 1999;24(21):2224-8.

32. Chesnut RM, Abitbol JJ, Garfin SR. Surgical management of cervical radiculopathy. Indication, techniques, and results. Orthop Clin North Am. 1992:23(3):461-74

33. van Jonbergen HP, Spruit M, Anderson PG, Pavlov PW. Anterior cervical interbody fusion with a titanium box cage: early radiological assessment of fusion and subsidence. Spine J. 2005:5(6):645-9.

34. Hilibrand AS, Balasubramanian K, Eichenbaum M, Thinnes JH, Daffner S, Berta $\mathrm{S}$, et al. The effect of anterior cervical fusion on neck motion. Spine (Phila Pa 1976). 2006;31(15):1688-92.

35. Shimamoto N, Cunningham BW, Dmitriev AE, Minami A, McAfee PC. Biomechanical evaluation of stand-alone interbody fusion cages in the cervical spine. Spine (Phila Pa 1976). $2001 ; 26(19): E 432-6$ 